\title{
Associations between Serotonergic Genes and Escitalopram Treatment Responses in Patients with Depressive Disorder and Acute Coronary Syndrome: The EsDEPACS Study
}

\author{
Hee-Ju Kang1, Kyung-Yeol Bae', Sung-Wan Kim¹, Il-Seon Shin1, Young Joon Hong², Youngkeun Ahn², \\ Myung Ho Jeong ${ }^{2}$, Sung-Woo Park ${ }^{3}$, Young-Hoon Kim³ ${ }^{3}$ Jin-Sang Yoon ${ }^{1}$, and Jae-Min Kim ${ }^{1 凶}$ \\ 'Department of Psychiatry, Chonnam National University Medical School, Gwangju, Republic of Korea \\ ${ }^{2}$ Department of Cardiology, Chonnam National University Medical School, Gwangju, Republic of Korea \\ ${ }^{3}$ Department of Neuropsychiatry, School of Medicine, Haeundae Paik Hospital, and Paik Institute for Clinical Research \& FIRST Research Group, \\ Inje University, Busan, Republic of Korea
}

\begin{abstract}
Genes related to serotonin are associated with responses to treatment for depression. We examined associations between the serotonin transporter (5-HTT) and serotonin 2a receptor (5-HTR2a) genes and responses to treatment for depressive disorders in acute coronary syndrome (ACS). A total of 255 patients who met the DSM-IV major or minor depressive disorder and recently developed ACS were randomly assigned to the escitalopram $(n=127)$ or placebo $(n=128)$ group in this 24-week double-blind trial (ClinicalTrial.gov identifier: NCT00419471). Remission was defined as a Hamilton Rating Scale for Depression (HAMD) score $\leq 7$. Assays were performed for the 5-HTTLPR, STin2 VNTR, 5-HTR2a 102T/C, and 5-HTR2a 1438A/G genotypes. Escitalopram was superior to placebo for treating depressive disorder with ACS but there were no significant associations between serotonergic genes and treatment responses even when considering ACS severity. The effect of escitalopram was independent of 5-HTT and 5-HTR2a polymorphisms.
\end{abstract}

Psychiatry Investig 2016;13(1):157-160

Key Words Acute coronary syndrome, Depression, Serotonin transporter, Serotonin receptor, Pharmacogenetic study.

\section{INTRODUCTION}

Depression is common with acute coronary syndrome (ACS) and is associated with increased morbidity and mortality. ${ }^{1}$ There is a great need for the treatment of depressive disorder in ACS patients, and a review of randomized controlled trials provided evidence of a small beneficial effect of selective serotonin reuptake inhibitors (SSRIs) compared with placebo. ${ }^{2}$ It would be helpful to find predictors of antidepressant response to improve treatment outcomes.

There is an emerging body of evidence that suggests that genetic factors substantially contribute to inter-individual variability in antidepressant responses. ${ }^{3}$ Serotonergic signaling at

Received: May 6, 2015 Revised: June 24, 2015

Accepted: June 26, 2015 Available online: November 20, 2015

$\triangle$ Correspondence: Jae-Min Kim, MD, PhD

Department of Psychiatry, Chonnam National University Medical School, 160 Baekseo-ro, Dong-gu, Gwangju 61469, Republic of Korea

Tel: +82-62-220-6143, Fax: +82-62-225-2351, E-mail: jmkim@chonnam.ac.kr

(c) This is an Open Access article distributed under the terms of the Creative Commons Attribution Non-Commercial License (http://creativecommons.org/licenses/bync/3.0) which permits unrestricted non-commercial use, distribution, and reproduction in any medium, provided the original work is properly cited. neuronal synapses is controlled by several pathways, including serotonin transporters and serotonin receptors. Therefore, various genes affecting serotonin functioning should be considered with respect to SSRI treatment responses. A meta-analysis of depression research found that the serotonin transporter genelinked promoter region (5-HTTLPR), a variable number of tandem repeats in 5-HTT intron 2 (STin2 VNTR), and serotonin 2a receptor (5-HT2Ra) polymorphisms may modulate responses to SSRIs. ${ }^{4}$ However, the associations between serotonergic genes and treatment responses have not been investigated in post-ACS depression despite the fact that there have been a number of randomized controlled trials involving SSRIs. ${ }^{2}$

Our study aimed to investigate whether 5-HTT and 5-HTR2a genotypes are associated with treatment responses in those with depression and ACS.

\section{METHODS}

This analysis was conducted during a 24 -week, doubleblind, placebo-controlled trial of escitalopram as part of the Escitalopram for DEPression in ACS (EsDEPACS) study 
(ClinicalTrial.gov: NCT00419471). ${ }^{5}$ Written informed consent was collected, and the study was approved by the Chonnam National University Hospital Institutional Review Board. Detailed descriptions of the study design and eligibility criteria have been published. ${ }^{6}$ The present 24 week double-blind trial included 300 patients who had recently developed ACS and were diagnosed with depressive disorder (major or minor) according to the DSM-IV criteria using the Mini-International Neuropsychiatric Interview (MINI). ${ }^{7}$ The patients were randomly assigned to either the escitalopram group $(n=149)$ or the placebo group ( $n=151)$ using a computer-generated randomization code provided by a statistician who was independent of the recruiting clinicians. The drugs were provided to the patients by pharmacists blind to the patients' status. Outcome measurement and adverse event monitoring were carried out by clinicians blinded to the treatment allocation. The primary efficacy measure was the Hamilton Depression Rating Scale (HAMD), ${ }^{8}$ and EsDEPACS determined that escitalopram was superior to placebo. ${ }^{5}$ Of 300 EsDEPACS participants, 255 agreed to blood assays, and the subjects evaluated at least once after baseline sample collection comprised the sample for this pharmacogenetic analysis. Data on sociodemographic characteristics, cardiovascular risk factors, and current cardiac status potentially associated with ACS treatment response were also obtained.

With respect to genotyping, polymerase chain reaction (PCR) methods were used for allele detection. The genotypes were delineated as ' $1 / \mathrm{l}$ ', ' $1 / \mathrm{s}$ ', and ' $\mathrm{s} / \mathrm{s}$ ' for the 5 -HTTLPR polymorphism, 'T/T', 'T/C', and 'C/C' for the 5-HTR2a 102T/C polymorphism, and 'G/G', 'G/A', and 'A/A' for the 5-HTR2a 1438A/G polymorphism. For the STin2 VNTR polymorphism, the 9 allele was extremely rare (present in only three participants), and thus the genotypes were categorized as ' $10 / 12$ ' and ' 9 or $12 / 12$ '.

The statistical analysis compared the baseline demographic and clinical characteristics of escitalopram and placebo groups using t-tests, $\chi^{2}$ tests, or Fisher's exact tests, as appropriate. Remission was defined as a HAMD score $\leq 7$. Remission status was recorded at each follow-up point. Achievement of remission was coded only when the remission was maintained until the 24-week study endpoint or the last follow-up examination. The allele frequencies for the four polymorphisms of those who did and did not achieve remission in the escitalopram and placebo groups were compared separately using $\chi^{2}$ tests. Finally, the interaction terms between treatment groups and genotypes were calculated using a multivariate logistic regression model with baseline covariates. Additional analyses were conducted with the same analytical methods according to ACS severity (Killip score of $\leq 1$ or $>1$ ) because serotonergic genes are known to predict the cardiac prognosis of a patient. ${ }^{9}$ Statistical analyses were performed using SPSS 18.0 software.

\section{RESULTS}

Of 255 participants (127 randomly assigned to the escitalopram group, 128 randomly assigned to the placebo group), 49 (19\%) left the study following baseline data collection, leaving 206 (104 in the escitalopram and 102 in the placebo groups) to

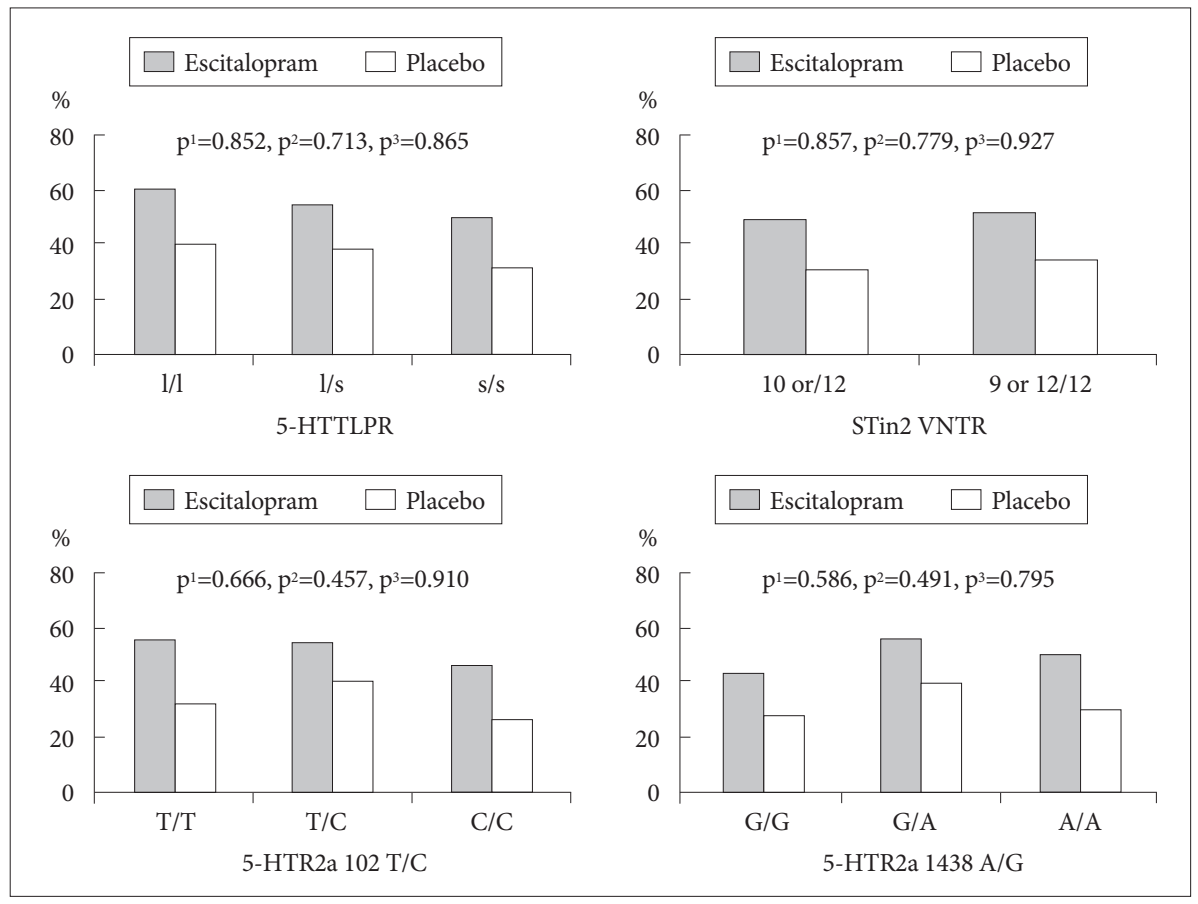

Figure 1. Hamilton Depression Rating Scale remission rates by treatment groups and genotypes. 5-HTTLPR: serotonin transporter gene linked promoter region, STin2 VNTR: serotonin transporter intron 2 variable number tandem repeat, 5-HTR2a: serotonin 2a receptor, $p^{1}$ : values for remission status in escitalopram group, $p^{2}$ : values for remission status in placebo group, $\mathrm{p}^{3}$ : values for interaction between treatment groups and genotypes. 
form the sample for pharmacogenetic analysis. Baseline characteristics are summarized in (Supplementary Table 1 in the online-only Data Supplement). There were no significant differences between the escitalopram and placebo groups for any characteristic (all p-values $>0.05$ ). Compared with patients available to follow-up, the Killip class $(\mathrm{p}=0.019)$ and serum CK-MB level $(\mathrm{p}=0.022)$ were significantly higher in patients sampled only at baseline, but no other significant differences were identified (all p-values $>0.05$ ).

The overall remission rate was significantly higher in the escitalopram group (51.9\%) compared with the placebo group $(34.3 \%)\left(\chi^{2}=6.507, \mathrm{p}\right.$-value $\left.=0.011\right)$. The remission rates are shown according to treatment group and genotype in Figure 1. Allele frequencies did not significantly differ with respect to remission status in either the escitalopram or placebo group (all p-values $>0.45$ ). There were no significant relationships between treatment groups and any genotype (all p-values $>0.79$ ). The analyses were repeated after categorizing the patients based on ACS severity, but no significant differences were observed in any of the models (Supplementary Table 2 in the online-only Data Supplement).

\section{DISCUSSION}

It was found that escitalopram was superior to placebo for the treatment depressive disorder with ACS, but there were no significant associations between any of the serotonergic genes and treatment responses during the 24-week randomized controlled trial, even when considering ACS severity.

Of the polymorphisms assayed, 5-HTTLPR has been the most widely evaluated in pharmacogenetic analyses, as the serotonin transporter (5-HTT) clears the neurotransmitter at the synaptic cleft and, in turn, has crucial role in determining serotonergic functioning. However, mixed results were found in pharmacogenetic analyses of depression in general populations. A meta-analysis of 33 studies, including 19 Caucasian and 11 Asian samples, concluded that the 5-HTTLPR 1 allele better predicted SSRI responses in Caucasian samples, but findings were less clear in Asian samples. ${ }^{10}$ More specifically, in Korean samples, the s allele has been reported to be associated with a better SSRI response. ${ }^{11,12}$ It has also been reported that the 5-HTTLPR 1 allele is associated with platelet activation and increases in thromboglobulin, ${ }^{13}$ and that it therefore predicts a poorer ACS prognosis. ${ }^{9}$ In summary, the association between 5-HTTLPR and treatment responses differ in an ethnicity-dependent manner and is also related to the complex effects of cardiac prognosis. Our findings supported the lack of an association between 5-HTTLPR and SSRI response and also reinforced the possibility that other sociodemographic and clinical variables influence the response to SSRI treatment in Asian groups. $^{10}$

Of the other polymorphisms assayed, another common variant of 5-HTT, STin2 VNTR and the 12-repeat alleles (STin2.12) have been reported to have an association with SSRIs, particularly in Asian groups. ${ }^{14}$ Among the serotonin 2a receptor (5-HTR2a) genes, which include two polymorphisms, an MspI polymorphic site at position 102T/C and a 1438A/G polymorphism in the promoter region, no association has been found between the 102T/C polymorphism and the efficacy of antidepressants for the treatment of general depression. ${ }^{14}$ The $\mathrm{G}$ allele of the $1438 \mathrm{~A} / \mathrm{G}$ polymorphism has been associated with a better antidepressant response in an Asian subsample. ${ }^{14}$ However, these conclusions are largely dependent on one or two studies. ${ }^{14}$ The role of these polymorphisms in the pathophysiology of ACS is unclear. In the middle of these mixed, controversial, and/or premature results, and considering the complex role of serotonergic genes on cardiac prognosis, ${ }^{9,13,15,16}$ it is difficult to conclude serotonergic pharmacogenetic associations with escitalopram in depressive patients with ACS.

Our study is the first evaluation of the association between serotonergic genes and treatment responses in those with depression with ACS using a randomized placebo-controlled pharmacogenetic approach. We conducted a comprehensive evaluation of several serotonergic genes. Recruitment was performed at a single site, which increased consistency in patient evaluation and treatment. Depressive disorder was diagnosed using a structured diagnostic interview, and all measurement methods for psychiatric and cardiovascular characteristics were well validated. Furthermore, a range of covariates were considered in the analyses. There were limitations in the pharmacogenetic analysis due to attrition, as $19 \%$ of the randomized subjects in the EsDEPACS trial left the study following baseline evaluation. Attrition was associated with a poorer baseline cardiac status. Therefore, ACS patients with more severe baseline pathology dropped out more frequently, which may have confounded the significance levels.

In general, there were no significant associations between serotonergic genes and escitalopram treatment responses, even when considering ACS severity, which has been associated with serotonergic genes. ${ }^{9}$ These negative findings suggest that the treatment response of patients with depression and ACS may be influenced by sociodemographic and clinical variables rather than serotonergic genes which differ from the treatment response of depression in general. In conclusion, the present findings do not provide any evidence to support the use of serotonergic genetic screening as a predictive factor for the treatment response to escitalopram or that it would benefit patients with depressive disorder and ACS. The beneficial effect of escitalopram on the treatment of depressive disorder with ACS 
was independent of 5-HTT and 5-HTR2a polymorphisms.

\section{Supplementary Materials}

The online-only Data Supplement is available with this article at http://dx.doi.org/10.4306/pi.2016.13.1.157.

\section{Acknowledgments}

This study was supported by grants of the Korea Health 21 R\&D, Ministry of Health and Welfare, Republic of Korea (HI12C0003), by Basic Science Research Program through the National Research Foundation of Korea (NRF) funded by the Ministry of Science, ICT and future Planning (NRF2013R1A2A2A01067367), and also by a grant (CRI15902-21) of Chonnam National University Hospital Biomedical Research Institute.

\section{REFERENCES}

1. Rudisch B, Nemeroff CB. Epidemiology of comorbid coronary artery disease and depression. Biol Psychiatry 2003;54:227-240.

2. Baumeister H, Hutter N, Bengel J. Psychological and pharmacological interventions for depression in patients with coronary artery disease. Cochrane Database Syst Rev 2011;9:CD008012.

3. Maier W, Zobel A. Contribution of allelic variations to the phenotype of response to antidepressants and antipsychotics. Eur Arch Psychiatry Clin Neurosci 2008;258(Suppl 1):12-20.

4. Kato M, Serretti A. Review and meta-analysis of antidepressant pharmacogenetic findings in major depressive disorder. Mol Psychiatry 2010; 15:473-500.

5. Kim JM, Bae KY, Jung BO, Kang HJ, Kim SY, Kim SW, et al. Escitalopram treatment for depressive patients with acute coronary syndrome: a 24 week double-blind placebo-controlled trial. J Clin Psychiatry 2015; 76:62-68.

6. Kim JM, Bae KY, Kang HJ, Kim SW, Shin IS, Hong YJ, et al. Design and methodology for the Korean observational and escitalopram treatment studies of depression in acute coronary syndrome: K-DEPACS and EsDEPACS. Psychiatry Investig 2014;11:89-94.
7. Sheehan DV, Lecrubier Y, Sheehan KH, Amorim P, Janavs J, Weiller E, et al. The Mini-International Neuropsychiatric Interview (M.I.N.I): the development and validation of a structured diagnostic psychiatric interview for DSM-IV and ICD-10. J Clin Psychiatry 1998;59(Suppl 20): 22-33.

8. Hamilton M. A rating scale for depression. J Neurol Neurosurg Psychiatry 1960;23:56-62.

9. Fumeron F, Betoulle D, Nicaud V, Evans A, Kee F, Ruidavets JB, et al. Serotonin transporter gene polymorphism and myocardial infarction: Etude Cas-Temoins de l'Infarctus du Myocarde (ECTIM). Circulation 2002;105:2943-2945.

10. Porcelli S, Fabbri C, Serretti A. Meta-analysis of serotonin transporter gene promoter polymorphism (5-HTTLPR) association with antidepressant efficacy. Eur Neuropsychopharmacol 2012;22:239-258.

11. Kim H, Lim SW, Kim S, Kim JW, Chang YH, Carroll BJ, et al. Monoamine transporter gene polymorphisms and antidepressant response in Koreans with late-life depression. JAMA 2006;296:1609-1618.

12. Bousman CA, Sarris J, Won ES, Chang HS, Singh A, Lee HY, et al. Escitalopram efficacy in depression: a cross-ethnicity examination of the serotonin transporter promoter polymorphism. J Clin Psychopharmacol 2014;34:645-648.

13. Whyte EM, Pollock BG, Wagner WR, Mulsant BH, Ferrell RE, Mazumdar S, et al. Influence of serotonin-transporter-linked promoter region polymorphism on platelet activation in geriatric depression. Am J Psychiatry 2001;158:2074-2076.

14. Niitsu T, Fabbri C, Bentini F, Serretti A. Pharmacogenetics in major depression: a comprehensive meta-analysis. Prog Neuropsychopharmacol Biol Psychiatry 2013;45:183-194.

15. Yamada S, Akita H, Kanazawa K, Ishida T, Hirata K, Ito K, et al. T102C polymorphism of the serotonin (5-HT) $2 \mathrm{~A}$ receptor gene in patients with non-fatal acute myocardial infarction. Atherosclerosis 2000;150: 143-148.

16. Coto E, Reguero JR, Alvarez V, Morales B, Batalla A, Gonzalez P, et al. 5-Hydroxytryptamine 5-HT2A receptor and 5-hydroxytryptamine transporter polymorphisms in acute myocardial infarction. Clin Sci (Lond) 2003;104:241-245. 